\title{
A Downlink Interference Suppression Scheme for Ultra Dense Networks
}

\author{
Yannan Wanga , Guoping Tan ${ }^{12}$ 3b Fei Feng $^{c}$ \\ HoHai University \\ Nanjing, 210000, China \\ E-mail: ${ }^{a} 1350962481 @ q q$. com; ${ }^{b}$ gptan@hhu. edu.cn; ${ }^{\circ} 623940918 @ q q \cdot c o m$
}

\section{Guomin $\mathbf{W} \mathbf{u}^{\mathrm{d}}$,Yueheng $\mathbf{L i}^{\mathrm{e}}$}

HoHai University

Nanjing, 210000, China

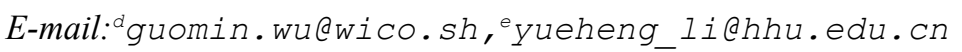

\begin{abstract}
In scenarios of the downlink of the ultra-dense network (UDN), in order to effectively overcome the influence of the interference sources of multiple similar-intensity on the user performance, resulting from the decrease of the distance between nodes and the similarity of the transmission loss of the neighboring nodes, a user-centric interference coordination scheme based on priority (PUCIC) was proposed in this paper.In order to facilitate quantifying the bandwidth performance gains achieved through base stations collaboration, a performance metric was proposed to calculate the bandwidth loss-gain-rate. Through the quantitative value of bandwidth loss-gain-rate, it's easy to know whether the user performance gain obtained in the scheme is less than the bandwidth loss to determine whether the scheme is profitable in specific UDN scenarios. Thus, parameter settings were optimized by the maximum loss ratio that the system can accept.
\end{abstract}

CENet2017

22-23 July 2017

Shanghai, China

\footnotetext{
${ }^{1}$ Speaker

${ }^{2}$ Correspongding Author

${ }^{3}$ This work was supported by Fundamental Research Funds for the Central Universities (2015B18914; 2014B33114), Key Laboratory of Wireless Sensor Network and Communication, Shanghai Institute of Microsystem and Information Technology, Chinese Academy of Sciences (2016001) and Graduate student scientific research innovation projects in jiangsu province (KYLX_0436).
} 


\section{Introduction}

Ultra-dense network (UDN) [1] is one of the key technologies of 5G mobile communication. It increases network density by increasing the deployment density of the lowpower site. Making the node closer to the end user greatly improves the system capacity., The spectrum efficiency and power efficiency are improved as well. However, due to the reduction of node spacing, the transmission loss of adjacent nodes is not much different. When the multiple interference sources are closer to the user, it makes the user more seriously interfered than the traditional cellular network. Therefore, how to solve the problem of performance loss caused by multiple interference sources and how to improve user performance with network cooperation and interference management become the key problems in 5G UDN interference suppression.

Traditionally, there are many ways to deal with inter-cell interference issues. Inter-cell interference suppression is divided into three types: inter-cell interference randomization [2], inter-cell interference cancellation [3] and inter-cell interference coordination/avoidance [4]. In the interference management, the traditional frequency reuse scheme is based on user classification which divides the users into central users and edge users, and the performance will be limited to the edge users. There is also an existing interference management method which divides the whole network into disjoint clusters, and the performance will be limited to cluster edge users [5-6] as well. However, in the 5G UDN network, the low-power nodes are densely distributed and have a small coverage area. Therefore, the traditional user-based and clusterbased interference managements are no longer applicable to 5G UDN.

This paper proposes a priority-based user-centric interference coordination (PUCIC) scheme which can improve the users' performance with the lowest possible complexity. The main bottleneck of the interference network, namely, the cell edge users, will be solved.

\section{System Model}

A super-dense network with inter-cell interference is taken as an exemple. As shown in Figure 1, the base station has $\mathrm{M}$ antennas while the user has a single antenna.

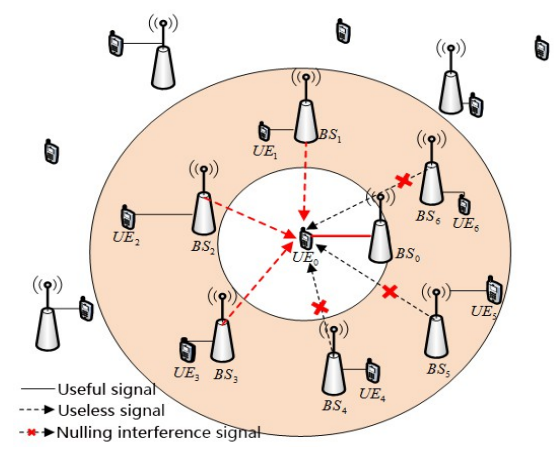

自

Figure 1:Super dense interference network diagram

The base station and the users in the cell are randomly distributed in a certain area. The number of the base stations is denoted as $L_{B S}$, where $L_{B S}=L+1$ and the 0th base station is the target base station. The other $\mathrm{L}$ base stations are the interfering base stations. The number of users is denoted as $\mathrm{K}$. The nearest base station is the user's local base station, while the other nearby base stations are regarded as interfering base stations. Let's set user $\mathrm{K}$ as the central user for analysis. In the downlink of the cellular communication network, the user is subjected to the interference signals from neighboring base stations. Thus, the signal received by the user $\mathrm{K}$ 
under the base station i, $y_{k, i}$, contains the useful signal of the local base station i, $s_{k, i}$, and the interference signals of adjacent base stations $\mathrm{j}\left(j=1, \ldots, L_{B S}\right.$ and $\left.j \neq i\right), I_{k, j}$. The received signal of the user $\mathrm{K}$ is expressed as:

$$
y_{k, i}=s_{k, i}+\sum_{j=1, j \neq i}^{L_{B S}} I_{k, j}+n
$$

Where $S_{k, i}=g_{k, i} h_{k, i} x_{k, i}$ is the desired signal of the user $\mathrm{K}$ at the base station $\mathrm{i}$, $I_{k, j}=g_{k, j} h_{k, j} x_{k, j} \quad$ represents interference signal from the base station $\mathbf{j}$ received by the user $\mathrm{K}$, and $\mathrm{n}$ is a white Gaussian noise. Here, $g_{k, i}=p_{i} d_{k, i}^{-\alpha} \sqrt{2}$ represents the large-scale fading of the base station $\mathrm{i}$ to the user $\mathrm{K}, \quad P_{i}$ is the transmission power of the base station $\mathrm{i}, d_{k, i}$ is the distance between the base station $\mathrm{i}$ and the user $\mathrm{K}, \alpha$ is the attenuation factor. $h_{k, i}$ represents the small-scale fading of the base station $\mathrm{i}$ to the user $\mathrm{K}$ and $x_{k, i}$ represents the transmission signal of the base station $i$.

In the PUCIC scheme, the user firstly ranks the received signal power $P_{i} \quad(i=1, \cdots, L)$ from the $\mathrm{L}$ interference base stations in descending order to obtain the priority of the interference coordination $I_{1}, I_{2} \ldots, I_{L}$.Through a series of processes upon the user judgments, the minimum $L_{1}$ and $L_{2}$ can be determined by the conditions which meet $S I N R \geqslant \gamma$. The number of base stations that cooperate with the local base stations $B S_{0}$ for the user will be set to zero with beamforming.

Here, it is assumed that $\Theta_{1}=\left\{B S_{1}, B S_{2}, B S_{3}\right\}$ is the set of base stations cooperate with the local base station and $\Theta_{2}=\left\{B S_{4}, B S_{5}, B S_{6}\right\}$ is the set to be nulled. The user's received signal is expressed as:

$$
y_{0}=s_{0}+\sum_{i \in \Theta_{1}} I_{0, i}+\sum_{j \in \Theta_{2}} I_{0, j}+\sum_{j \in \Psi_{I C B S}-\Theta_{1}-\Theta_{2}} I_{0, j}+n
$$

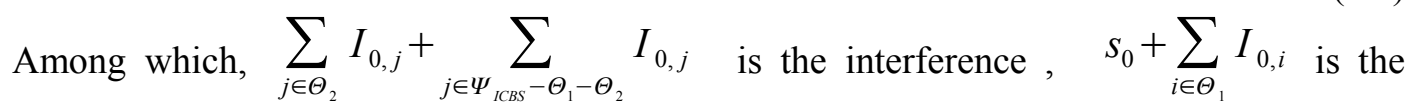
useful signal received by the user. $\sum_{j \in \Theta_{2}} I_{0, j}$ is set to zero after the local base station beamforms. Thus the user's received signal is expressed as:

$$
y_{0}=s_{0}+\sum_{i \in \Theta_{1}} I_{0, i}+\sum_{j \in \Psi_{I C B S}-\Theta_{1}-\Theta_{2}} I_{0, j}+n
$$

At this time, the strong interference around the user $U E_{0}$ is converted into a useful signal, and the second strong interference is suppressed.

\section{PUCIC Mechanism}

\subsection{PUCIC Process}


Based on the user-centered idea, the PUCIC determines the number of base stations $L_{1}$ to be cooperated with the local base station and the number of base stations $L_{2}$ that are interference nulled by the local base station. PUCIC suppresses inter-cell interference and improves user performance and system with the base station collaboration and beamforming.

The PUCIC program consists of three execution modules and details are as follows:

\subsubsection{Generate Coordination Priorities}

The user first obtains the priority order of the interference coordination according to the received signal power $P$ from the surrounding $L$ interfering base stations $\Phi=\left\{I_{1}, I_{2}, \ldots, I_{L}\right\}$.

\subsubsection{User Judgment Process}

The corresponding SINRs are sequentially traversed and estimated according to the coordination priority and the minimum $L_{1}$ and $L_{2}$ should satisify the conditions of SINR $\leqslant \gamma \quad\left(\gamma\right.$ is the threshold required for the minimum SINR of the user service). $L_{1}$ is the number of elements $\Theta_{1}$ of the base stations set together with the local base station $B S_{0}$ for the user's cooperation. is the number of elements $\Theta_{2}$ of the base stations set to null at the local base station $B S_{0}$.

\subsubsection{Collaborative Transmission And Interference Nulling Process}

The $L_{1}$ cooperative base stations and the $L_{2}$ interference nulling base stations carry out the corresponding cooperative transmission and interference nulling for local users respectively . CoMP is used by PUCIC for joint transmission (JT) and cooperative scheduling / beamforming CS / CB.

\subsection{User Decision Algorithm}

SINR estimation formula for the user is $\operatorname{SINR}=\frac{P_{0}+\sum_{i=1}^{L_{1}} P_{i}}{\sum_{j=L_{1}+L_{2}+1}^{L} P_{j}+\sigma^{2}}$, and the specific steps of the user's decision are as follows:

User decision calculation $L_{1} 、 L_{2}$ algorithm is based on the UDN scene. In the UDN, there are more intensive base stations. The implementation of a case of $L_{1}$ and $L_{2}$ decision algorithm is on the condition of $M \leqslant L$. With regards tosetting $M \leqslant L$, the specific descriptions are as follows:

1. Let $L_{1}=0$, the user $U E_{0}$ estimates SINR at $L_{2}=1$ and verifies whether the judgment condition $\operatorname{SINR} \leqslant \gamma$ is satisfied. If it is satisfied, $L_{2}=1$ is the request; if it is not satisfied, follow Step 1-1 method (below) : traverse $L_{2}$ and estimate $S I N R_{1}$ until you find the first $L_{2}$ that satisfies $\operatorname{SINR}_{1}^{(n)} \geqslant \gamma \quad$ (where $n \leqslant M-1$ ). According to the minimum requirements to meet user business on the basis of the principles of complexity, $L_{1} 、 L_{2}$ are for the request; 
1-1 Method:1-1a. Fix $L_{1}$ constant to determine whether it will meet $L_{1}+L_{2}+1 \leqslant L$. If it's not satisfied, then jump to Step 2. If satisfied, then make $L_{2}=1$, predict $S I N R_{1}$ and mark $\operatorname{SINR}_{1}^{(1)}$. If it satisfies $\operatorname{SINR}_{1}^{(1)} \geqslant \gamma$, then $L_{2}=1$ is the request, otherwise continue to the next step;

1-1b. Repeat this step until you find the first one $L_{2}$ satisfies $\operatorname{SINR}_{1}^{(n)} \geqslant \gamma \quad$ (where $n \leqslant M-1 \quad$ ). Then this $L_{2}$ is the optimal one for $L_{1}$.

2. Let $L_{1}=1$, the user $U E_{0}$ estimates $\operatorname{SINR}_{2}$ of $L_{2}=M-1$, verifies whether it will meet the decision condition $\operatorname{SINR}_{2} \geqslant \gamma$. If it's not satisfied, then jump to step 3; if satisfied, according to the method of step 1-1, traverse $L_{2}$ andestimate $S_{N N R_{2}}$ until it meets $\operatorname{SINR}_{2}^{(n)} \geqslant \gamma \quad$ (where $n \leqslant M-1 \quad$ ) the first $L_{2}$ so far. Then the $L_{1}$ and $L_{2}$ are the best. In addition, in the process of traversing $L_{2}$ adering to the method of step 1-1, $L_{2}+1$ should be satisfied under the condition that it satisfies $L_{1}+L_{2}+1 \leqslant L$. Skip to Step 3 if the condition is not satisfied;

3. Under the condition that it satisfies $L_{1}+1 \leqslant L$, let $L_{1}+1$ repeat this step until the first $L_{1}$ in $L_{2}=M-1$ and $S I N R_{c} \geqslant \gamma$ are satisfied.Traverse $L_{2}$ following the method of step 1-1 to find the first $L_{2}$ that satisfies $\operatorname{SINR}_{c}^{(n)} \geqslant \gamma \quad$ (where $c \leqslant L, n \leqslant M-1, c+n \leqslant L$ ). Then $\quad L_{1}$ and $L_{2}$ are for the request. Furthermore, if the process of $L_{1}+1$ does not meet $L_{1}+1 \leqslant L$, then the $L_{1}$ and $L_{2}=M-1$ are optimal. In the process of traversing $L_{2}$ in step $1-1, \quad L_{2}+1$ shouldbe satisfied under the condition of satisfying $L_{1}+L_{2}+1 \leqslant L$, and if the condition is not satisfied, $L_{1} 、 L_{2}$ are for the request;

4.On the basis of $L_{1}$ in Step 3, if the algorithm does not satisfy $L_{1}+1 \leqslant L$, the algorithm ends and the $L_{1}$ and $L_{2}$ obtained in Step 3 are optimal. If $L_{1}+1 \leqslant L$ is satisfied, then $L_{1}+1$, traverse $L_{2}$ in the way of Step 1-1, and estimate $\operatorname{SINR}_{c+1}$ until first $L_{2}$ satisfying $\operatorname{SINR}_{c+1}^{(n)} \geqslant \gamma$ (where $c \leqslant L, n \leqslant M-1, c+n \leqslant L$ ) is found. Besides, in the process of traversing $\quad L_{2}$ according to the method of step 1-1, $L_{2}+1$ should be satisfied under the condition that it satisfies $\quad L_{1}+L_{2}+1 \quad L \quad$.The algorithm ends if the condition is not satisfied.

5. Compare $\operatorname{SINR}_{c}^{(n)}$ and $\operatorname{SINR}_{c+1}^{(n)}$. If it satisfies $\left|S I N R_{c+1}^{(n)}-\operatorname{SINR} R_{c}^{(n)}\right| \leqslant \zeta$, then select the $L_{1}$ and $L_{2}$. Corresponding $\operatorname{SINR}_{c}^{(n)}$ is the optimal; otherwise select the $L_{1}$ and $L_{2}$ and corresponding $\operatorname{SINR}_{c+1}^{(n)}$ is the optimal.

\section{PUCIC PerformanceSimulation Analysis}

In order to simulate the impact of PUCIC mechanism on user SINR under different parameter changing environment, this paper builds the corresponding UDN scene based on MATLAB platform. The performance indicators used to evaluate the PUCIC mechanism are user average signal-to-noise ratio (SINR), an average bandwidth loss rate.

\subsection{Comparison of Performance under Different Base Stations}


In order to analyze the user SINR gain obtained by coordinating the priority of the scheme and the base station, this paper compares the selection scheme UCIIC.,It is comparable because UCIIC does not use the base station cooperative transmission. The number of base stations BS_NUM of 10 and 30 is set. The number of antennas M is 4 .

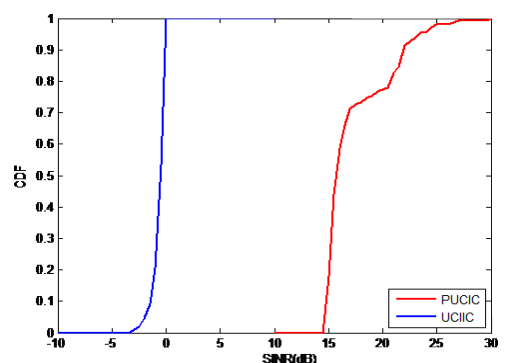

(a) when BS_NUM=10, PUCIC VS. UCIIC

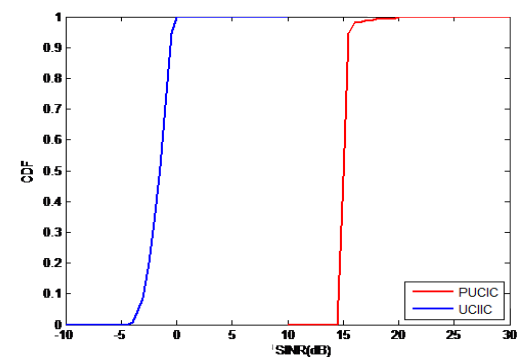

(b) when BS_NUM=30, PUCIC VS. UCIIC

Figure2:Comparison of User SINR CDF for Different Mechanisms when Base Station Number Changes

In Figure 2, the SINR of the two mechanisms decreases whenthe base station increases. The user SINR of the PUCIC is $15 \mathrm{~dB}$ higher than the UCIIC. When the number of base stations increases, PUCIC user SINR value is more concentrated. When the user SINR performance difference decreases, it, to a certain extent, enhances the user fairness.

\subsection{Comparison of Performance under DifferentAntenna Numbers}

When the number of base stations is constant, the more antennas there are the more interference each local base station can handle and the better performance the user can achieve. The number of antennas of 2 and 16 is set respectively. The number of base stations BS_NUM is 30 .

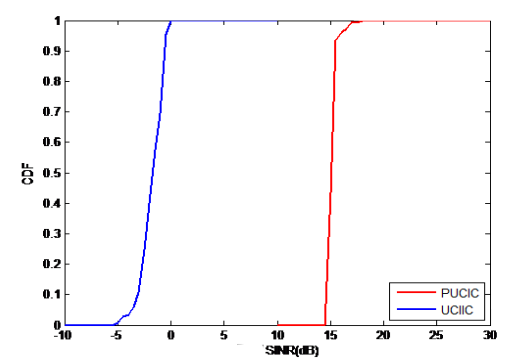

(a) when $\mathrm{M}=2$, PUCIC VS. UCIIC

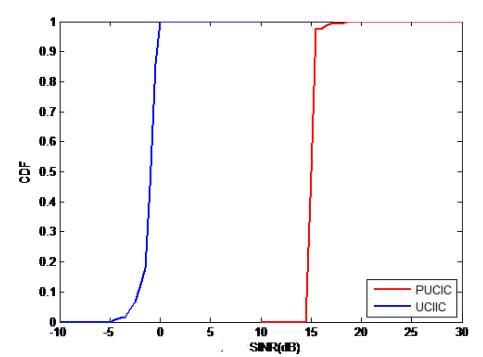

(b) when $\mathrm{M}=16$, PUCIC VS. UCIIC

Figure 3:Number of Antennas vs. User SINR CDF

In Figure3, the SINR of the two mechanisms does not change greatly with the increase of the antennas, and the user SINR of the PUCIC is $15 \mathrm{~dB}$ higher than the UCIIC. This indicates that the user SINR performance of the PUCIC is not limited by the number of base station antennas. In general, the increase in the number of antennas hardly affects the user SINR.

\subsection{Effects of Different Base Stations on PUCIC Bandwidth Performance}

In order to evaluate the performance improvement of the user performance obtained by the PUCIC and the bandwidth loss caused by the cooperation with the base station, the change of the average bandwidth loss-gain-rate of the PUCIC is observed when the number of base stations changes. BS_NUM, the number of base stations, is set as 10 and 30 . 
As shown in Figure 4, the PUCIC average bandwidth loss-gain-rate curves when the BS_NUM changesin the base stations. If the number of base stations is in the moderate, say 10 to 20, the loss ratio is acceptable, and PUCIC is effective and in the value of use., It will also be of a certain detection significance in the specific application.

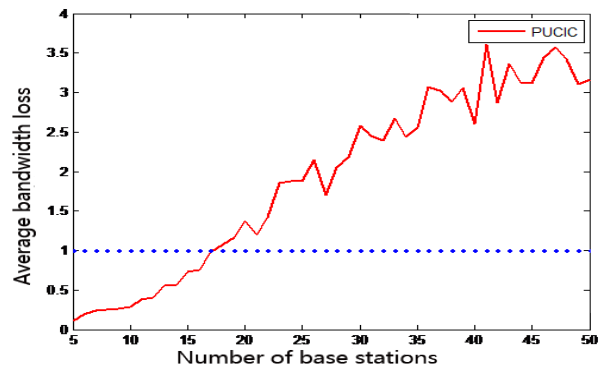

Figure 4:Effect of Base Station Number Change on PUCIC Average Bandwidth Deficit Rate

\section{Conclusion}

In this paper, a user-centric inter-cell interference suppression scheme, PUCIC, is proposed based on the base station collaboration for the inter-cell interference problem of UDN downlink. The scheme is proposed from the perspective of user performance experience. It is found that the PUCIC not only has a better user SINR performance than the UCIIC with achangable number of base stations it also effectively improves the user of the UCIIC SINR performance limited by the number of antennas and makes SINR about $15 \mathrm{~dB}$ gain. Moreover, , PUCIC effectively maintains the user fairness, which solved the problem of low performance limited to the existed traditional interference suppression mechanism. Taking the bandwidth loss rate into consideration, we can see that, on the whole, PUCIC improves the user performance and maintains the user fairness at the cost of the bandwidth loss.

\section{References}

[1] X. Ge, S. Tu, G. Mao, et al. 5G Ultra-Dense Cellular Networks[J]. Computer Science, 2015, 23(1):72-79

[2] Nikopour H, Baligh H. Sparse code multiple access[C]. Personal Indoor and Mobile Radio Communications (PIMRC), 2013 IEEE 24th International Symposium on. IEEE, 2013:332-336

[3] Zhai G W, Tian L, Zhou Y Q, et al. Load diversity based optimal processing resource allocation for super base stations in centralized radio access networks[J]. Sciece China Information Sciences, 2014, 57(4):1-12

[4] Liu J, Love R, Stewart K, et al. Design and Analysis of LTE Physical Downlink Control Channel[C]. Vehicular Technology Conference, 2009. VTC Spring 2009. IEEE 69th. IEEE, 2009:15

[5] Huang K, Andrews J G. An Analytical Framework for Multi-Cell Cooperation via Stochastic Geometry and Large Deviations [J]. IEEE Transactions on Information Theory, 2012, 59(4):25012516

[6] Zhang J, Chen R, Andrews J G, et al. Networked MIMO with clustered linear precoding[J]. IEEE Transactions on Wireless Communications, 2009, 8(4):1910-1921 